Musées, Patrimoine et Culture scientifiques et techniques

$192 \mid 2020$

novembre-décembre 2020

\title{
La visite dont vous êtes le héros
}

Denise Davin

\section{OpenEdition \\ Journals}

Édition électronique

URL : https://journals.openedition.org/ocim/4141

DOI : $10.4000 /$ ocim.4141

ISSN : 2108-646X

Éditeur

OCIM

Édition imprimée

Date de publication : 1 novembre 2020

Pagination : 8-15

ISSN : 0994-1908

Référence électronique

Denise Davin, «La visite dont vous êtes le héros », La Lettre de I'OCIM [En ligne], 192 | 2020, mis en

ligne le 01 novembre 2021, consulté le 11 décembre 2021. URL : http://journals.openedition.org/ocim/

4141 ; DOI : https://doi.org/10.4000/ocim.4141

Ce document a été généré automatiquement le 11 décembre 2021.

Tous droits réservés 


\section{La visite dont vous êtes le héros}

\section{Denise Davin}

Composition collective sur un thème dans le cadre d'une visite scolaire.

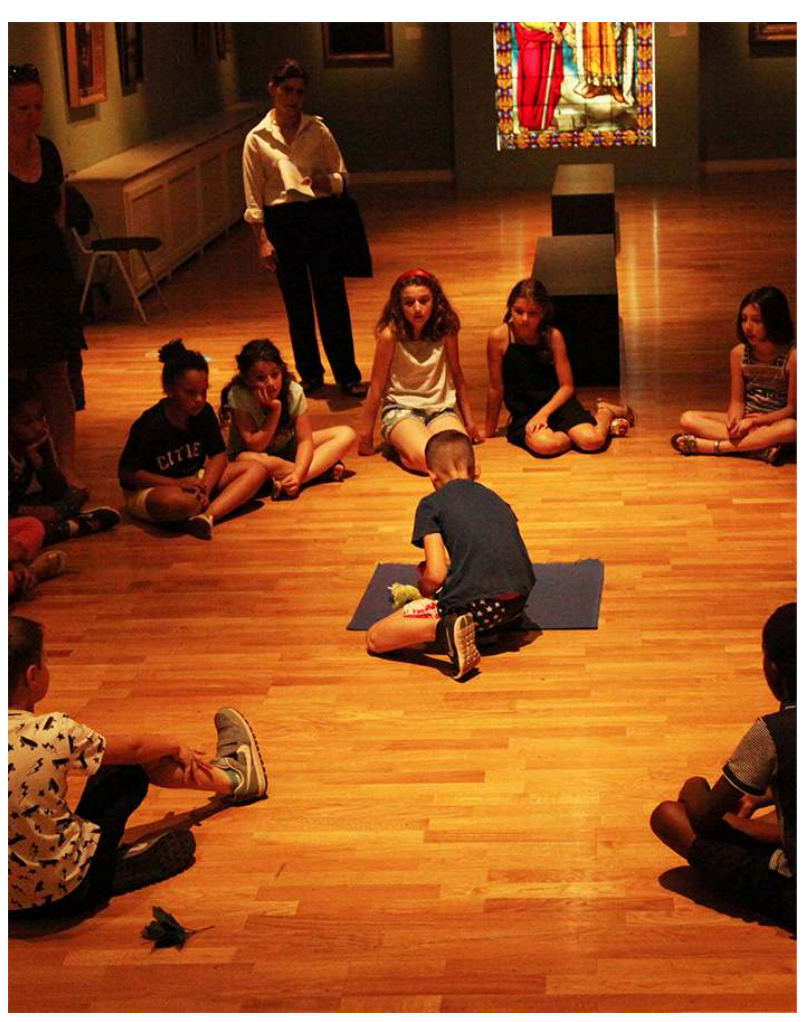

(c) L. Kieffer/Musée de La Cour d'Or - Metz Métropole 


\section{S'inspirer des préconisations et articles de référence pour construire son projet}

1 Les articles, enquêtes et rapports parus ces dernières années (et évoqués dans la suite du texte) autour de la médiation et des documents jeunes publics apportent des préconisations utiles à la conception de visites. Elles s'appliquent à tous types de musées comme celui de La Cour d'Or, alliant collections gallo-romaines, médiévales et Beaux-Arts dans un même bâtiment. Le travail mené en 2018-2019 lors des activités pédagogiques complémentaires (APC) prend en compte l'ensemble de ces réflexions pour susciter l'intérêt de l'enfant et lui faire partager de nouvelles émotions.

2 La conception des musées d'arts et d'histoire, d'après le ressenti des enseignants, a évolué. En 1998, une partie d'entre eux considérait le musée comme un lieu mort. D'autres, à l'opposé, l'imaginaient comme un lieu de loisirs développant une stratégie marketing dans l'objectif d'augmenter les chiffres de fréquentation. L'école demeurait, à leur sens, seule responsable de la transmission des savoirs ${ }^{1}$ ainsi qu'un lieu privilégié d'éveil et de développement de la sensibilité. Une transformation progressive des mentalités s'est opérée vers une adhésion aux musées dans le but, entre autres, de sortir du cadre scolaire ou de découvrir des objets authentiques.

3 Les rapports édités par le ministère de la Culture Évaluation des effets des actions de médiation culturelle sur les enfants et les adolescents (2016-2018) ${ }^{2}$ dressent un portrait des enseignants actuels et soulignent les effets constatés dans le domaine du péri éducatif. Ils sont devenus de véritables piliers pour ces projets et leur bon fonctionnement car, outre leur motivation, ils assurent la continuité entre l'activité au musée et l'école, notamment en travaillant sur des prolongements en classe. Enseignants et musées collaborent plus facilement. Il devient possible d'échanger des idées, de monter ensemble des projets à moyen, voire long terme.

Du point de vue de l'enfant, une enquête réalisée en $2015^{3}$ sur un public âgé de 7 à 12 ans, a montré que, lorsqu'il n'est pas expert, l'enfant souffre d'un a priori négatif des musées décrits comme un lieu " ennuyeux, fatiguant et silencieux ». La passivité latente dans les visites «subies » est aussi dénoncée par A. Van Dorpe et L. Scamps ${ }^{4}$. Afin de transformer ces freins en leviers, des solutions existent : travailler sur l'aspect ludique, développer le sens de l'observation, permettre d'expérimenter le musée en proposant des livrets spécifiquement conçus pour eux. Pour fonctionner, ces documents doivent se distinguer des livrets scolaires formels. Une expérience positive développe chez l'enfant, un souvenir plaisant à renouveler.

\section{Définition succincte des Activités pédagogiques complémentaires (APC) \\ "Instaurées dans le cadre de la réforme de l'organisation de la journée et de la semaine scolaires dans le premier degré, les APC font partie des obligations de service des professeurs qui les organisent et les mettent en cuvre dans toutes les écoles. Elles ne relèvent pas du temps d'enseignement obligatoire pour les élèves, mais s'ajoutent aux 24 heures hebdomadaires d'enseignement dues à tous et nécessitent l'accord des parents concernés. Leur organisation prend en compte l'offre péri-éducative existante, le cas échéant dans le cadre du projet éducatif territorial (PEDT).»}


Bulletin officiel de l'Éducation nationale ${ }^{\circ} 6$ du 7 février 2013, circulaire n²013-017 du 6 février 2013

\section{Application des préconisations aux APC du musée de La Cour d'Or}

Le service des publics du musée de La Cour d'Or - Metz Métropole conduit depuis 2012 différents projets d'APC. En 2019, onze thématiques différentes sont proposées permettant une approche transversale du musée. Les APC sont reconduits avec les écoles de proximité d'années en années. Une classe est divisée en petits groupes de 5 à 7 élèves. Le musée accueille des enfants du premier degré de la maternelle au CE2. Ils peuvent profiter du parcours permanent en fin d'après-midi avec leurs enseignants, une heure durant. Dans ce contexte, les élèves bénéficient d'un format optimal pour développer une relation personnelle avec les œuvres. Les règles sont clairement établies dès le départ : interdiction de courir ou de toucher. Des habitudes se créent au fil des visites.

6 Avec l'aide du médiateur, les enfants parviennent à vaincre leur peur de l'institution muséale. Ils s'approprient le lieu en se créant des repères spatiaux. Leur taux de participation s'accroît au cours de l'année et du vocabulaire est acquis. Les enfants verbalisent en tenant compte des règles de prises de paroles. Cette démarche est en adéquation avec le socle commun de connaissances, de compétences et de culture du Bulletin officiel ${ }^{5}$. La conception de l'activité prend également en compte la journée complète passée à l'école et le temps de trajet jusqu'au musée. L'apprentissage doit rester suffisamment léger et rythmé pour que l'enfant ne s'épuise pas.

\section{Une visite co-créative}

Le service des publics a conçu et mis en place un nouveau type de visite inédite qui immerge les enfants dans une histoire co-créative et originale ${ }^{6}$ à partir des collections présentées : Le musée dont vous êtes le héros. Elle s'inspire des livres d'aventures Le livre dont vous êtes le héros ${ }^{\circ 7}$ avec lesquels nombre d'enfants ont abordé la lecture de façon ludique, en devenant un vigoureux combattant ou un mage redouté. Initialement prévus pour un public d'adolescents, puis élargis, ces ouvrages sont à visée populaire, comme le souligne B. Solinski ${ }^{8}$. Aussi appelés livres-jeux, ils fonctionnent à partir de paragraphes numérotés suivant une arborescence. Chaque action renvoie vers un autre paragraphe et entraîne une conséquence différente. Dans ce type de projets, le titre de l'animation est important car il exerce une certaine attractivité sur le prescripteur (l'enseignant). 
SCHÉMA NARRATIF DU CONTE

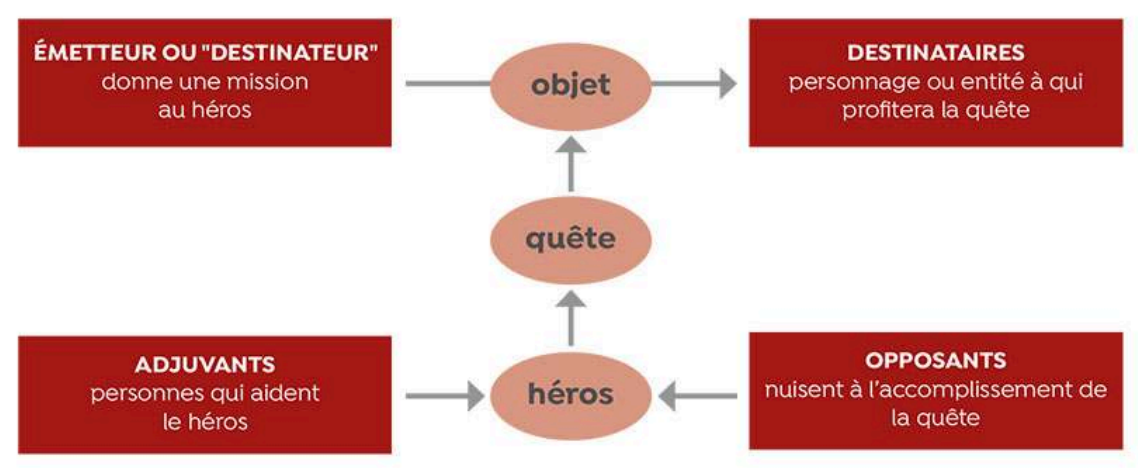

(c) D. Davin

\section{Genèse et développement du projet}

8 Ce projet émane d'une demande spécifique d'enseignants de CE1-CE2 ayant déjà participé à un premier APC thématique : Il était une fois, une construction de conte à l'aide d'œuvres. Il a été décidé de poursuivre l'expérience l'année suivante. Les élèves de CE1 étant susceptibles de revenir au musée en CE2, il était important de ne pas leur faire revivre la même chose. Une alternative a été envisagée. Dans le cahier des charges, la trame incluait une création d'histoire à partir des œuvres du musée. Elle devait reprendre le schéma narratif du conte, pan de la littérature jeunesse (ci-dessus).

Une animation s'appuyant sur le livre-jeu est apparue comme une bonne idée potentielle. Il s'agissait d'amener les enfants à construire une histoire en trois séances, ponctuée de choix mais aussi de hasard, grâce à ce support rarement exploité. L'analyse de l'existant ne nous a pas permis de trouver un projet similaire au moment de la conception, finalisée en février 2018. Toutefois le succès de L'exposition dont vous êtes le héros inaugurée en novembre 2018 à Yverdon en Suisse ${ }^{9}$ nous a confortés dans notre entreprise.

10 Une question essentielle s'est posée d'emblée : l'adaptation du concept de livre-jeu à un musée est-elle possible et comment?

11 En prenant pour sujet de test le Tournoi de tous les dangers ${ }^{10}$, récit des péripéties d'un jeune chevalier, notre équipe a détecté trois problèmes qui ont été résolus.

12 - le caractère individualiste du livre-jeu disparaît grâce à une lecture commune et des choix concertés.

- la liberté apparente, mais en réalité bridée par le texte, est réintroduite grâce à l'improvisation du médiateur.

14 - l'impasse de la mort du héros, obligeant le lecteur à recommencer du début, a été évitée en se muant en une simple perte de connaissance de quelques heures symboliques.

15 Pour nos besoins, le livre de récit illustré à choix multiples a été rédigé en amont. Il fonctionne par un système de numérotation et de renvois. Ce document sert de support à la création de l'histoire mais il fournit aussi au médiateur les indications essentielles pour mener la visite, comme noter le nom du héros sur la fiche de personnage ou 
observer une œuvre particulière. Ces éléments sont absents des livres originaux et sont une création spécifique d'aide à la visite. Dans le processus mis en place, les œuvres présentées dans le parcours permanent du musée servent à développer l'histoire et sont détournés à des fins imaginaires.

\section{La création des outils de médiation}

16 Le matériel utilisé à l'usage des visites a été fabriqué par le service des publics. S'agissant d'une phase test et d'expérimentation, les images et livrets ont été réalisés avec des dessins téléchargés sur les sites du domaine public ou des sites internet libres d'utilisation et de commercialisation avec, parfois, la demande de confirmation des droits d'utilisation de l'auteur. La carte du monde est dessinée sur un papier vieilli. La couverture du journal est fabriquée grâce à une projection d'encre et de sel, le tout est relié avec un fil de laine. Les enfants comme les enseignants étaient enchantés du résultat. Une externalisation visant à rationaliser la production des documents et améliorer leur graphisme est prévue à l'avenir car le public actuel est habitué à des rendus professionnels : une attente en partie due au poids grandissant que prennent l'image et l'audiovisuel dans notre quotidien.

Carte mentale simplifiée des choix et des rencontres possibles.

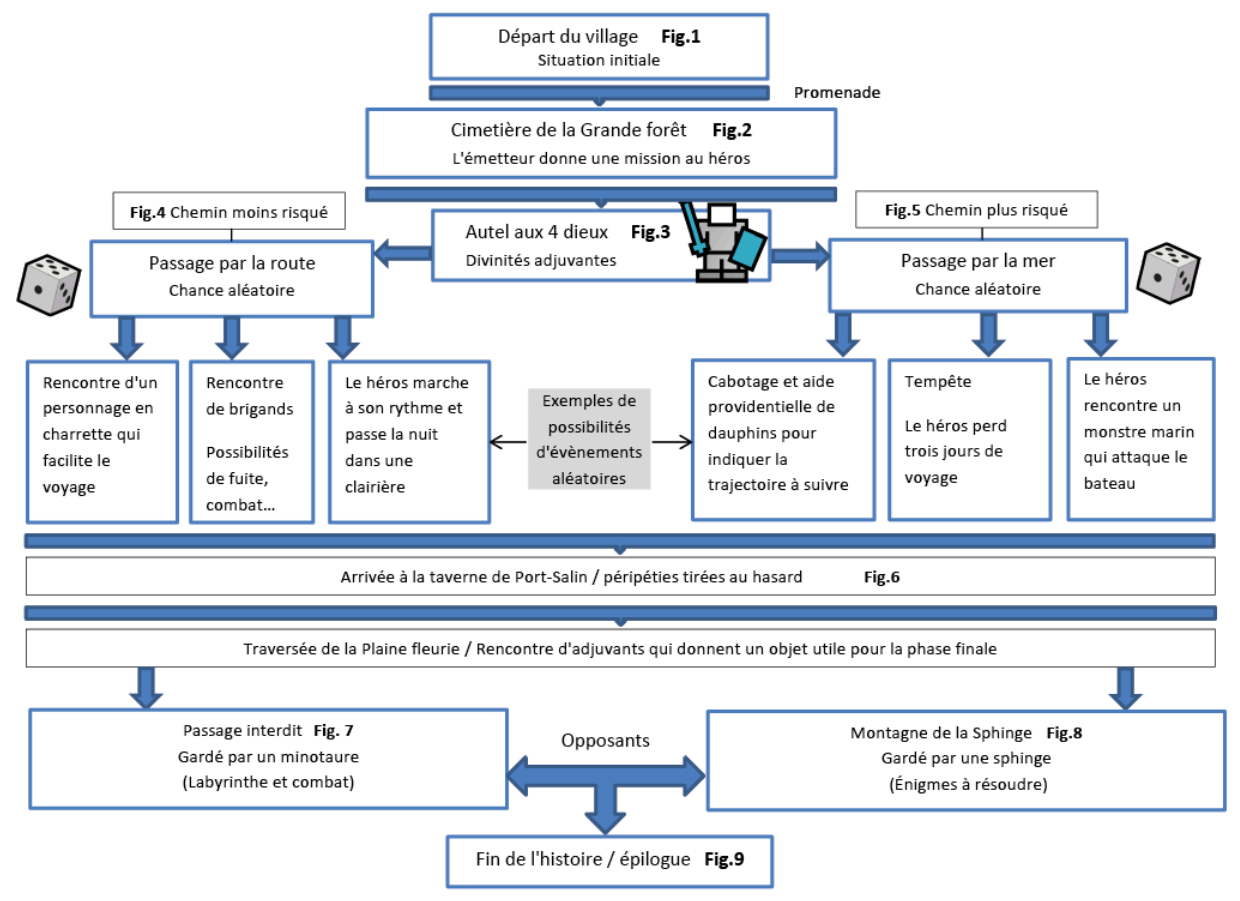

(C) D. Davin 


\section{La construction d'une histoire en trois séances}

\section{Première étape : introduction et création du héros}

Durant la première séance, les enfants créent leur futur héros ou héroïne d'un commun accord. Comme dans un conte, celui-ci grandit dans un village paisible qu'ils nomment (voir figure 1 du schéma ci-dessus). Lors d'une promenade, un élément perturbateur le pousse à accepter une quête qui l'entraine loin de chez lui (figure 2).

Une première moitié de carte à compléter est confiée à un élève. Cela le responsabilise. Les sculptures, tableaux et différents objets de nos collections sélectionnés pour l'aventure sont insérés sous forme de dessins schématisés dans cette carte imaginaire de façon à intégrer l'histoire.

Les œuvres observées finement avec le médiateur servent à l'intrigue. Par exemple les quatre dieux gréco-romains figurés sur un autel de pierre sont présentés comme des divinités adjuvantes (figure 3). Ils prêtent un attribut au choix pour aider le personnage dans sa quête. Les enfants s'initient, par ce moyen, à la mythologie gréco-romaine et enrichissent leur culture personnelle, notre objectif.

Un moment de pause permet de coucher par écrit les informations et de varier l'activité pour conserver l'attention des élèves. Chacun reçoit une fiche de personnage et peut illustrer et colorier son héros à sa guise, à condition de respecter les choix retenus collectivement et de faire figurer le bon attribut. Le dessin favorise la créativité et l'imagination de chacun. Un livret individuel en partie pré-rempli intitulé Journal d'aventurier est complété. Cette solution a été privilégiée pour des raisons de limite de temps. Les mots à l'orthographe compliquée sont écrits sur un tableau effaçable pour faciliter le travail des plus jeunes (CE1). Ce livret récapitulatif est distribué à la fin de chaque séance. À l'issue de celles-ci les enfants les conserveront, prolongeant ainsi leur expérience au musée.

Objectifs et effets des Activités pédagogiques complémentaires (APC)

Objectifs visés :

- enrichir la culture patrimoniale des élèves

- faire acquérir les compétences fondamentales en maîtrise de la langue

- tisser des liens étroits entre le musée, l'école et les familles

- réduire les inégalités scolaires

Effets constatés :

- travail en équipe des enfants et des enseignants

- climat de collaboration

- sensibilisation au musée

- réinvestissement 
- impact sur les parents et capacité à s'ouvrir aux musées

- plaisir des élèves

\section{Deuxième étape : l'aventure se poursuit au gré des rencontres}

Les enfants décident à chaque nouvel évènement du chemin à suivre, en prenant ou non des risques (figures 4 et 5). En lançant un dé à vingt faces qui introduit une part de hasard, les élèves font des rencontres aléatoires. Elles sont illustrées par des thèmes traités dans les œuvres. Puissant, malin ou intelligent, le héros possède des forces et des faiblesses dont il faut tenir compte. Si le groupe doit affronter un monstre, à lui d'utiliser à bon escient les compétences et attributs de son personnage en étant force de proposition. Le médiateur accepte toute solution qui paraît adéquate et improvise. De cette manière, l'histoire, vécue comme une aventure personnelle, les motive vraiment. Pour fonctionner, elle doit rester dynamique dans les phases d'action. La théâtralisation du médiateur est un atout essentiel pour la réussite de la séance. Les enfants sont d'autant plus volontaires pour avancer et explorer de nouveaux espaces que cette découverte passe par le jeu. Ils deviennent demandeurs et acteurs de leur visite.

Le héros ou l'héroïne fait une halte à la taverne imaginaire (figure 6) suggérée au musée par une reconstitution d'une chambre avec du mobilier du XIX ${ }^{\mathrm{e}}$ siècle. Il découvre dans cet endroit la seconde partie de la carte lui permettant de continuer son périple à travers les salles des différentes époques. Les enfants acquièrent ainsi une vision globale des collections.

Les œuvres finement observées avec le médiateur servent à l'intrigue.

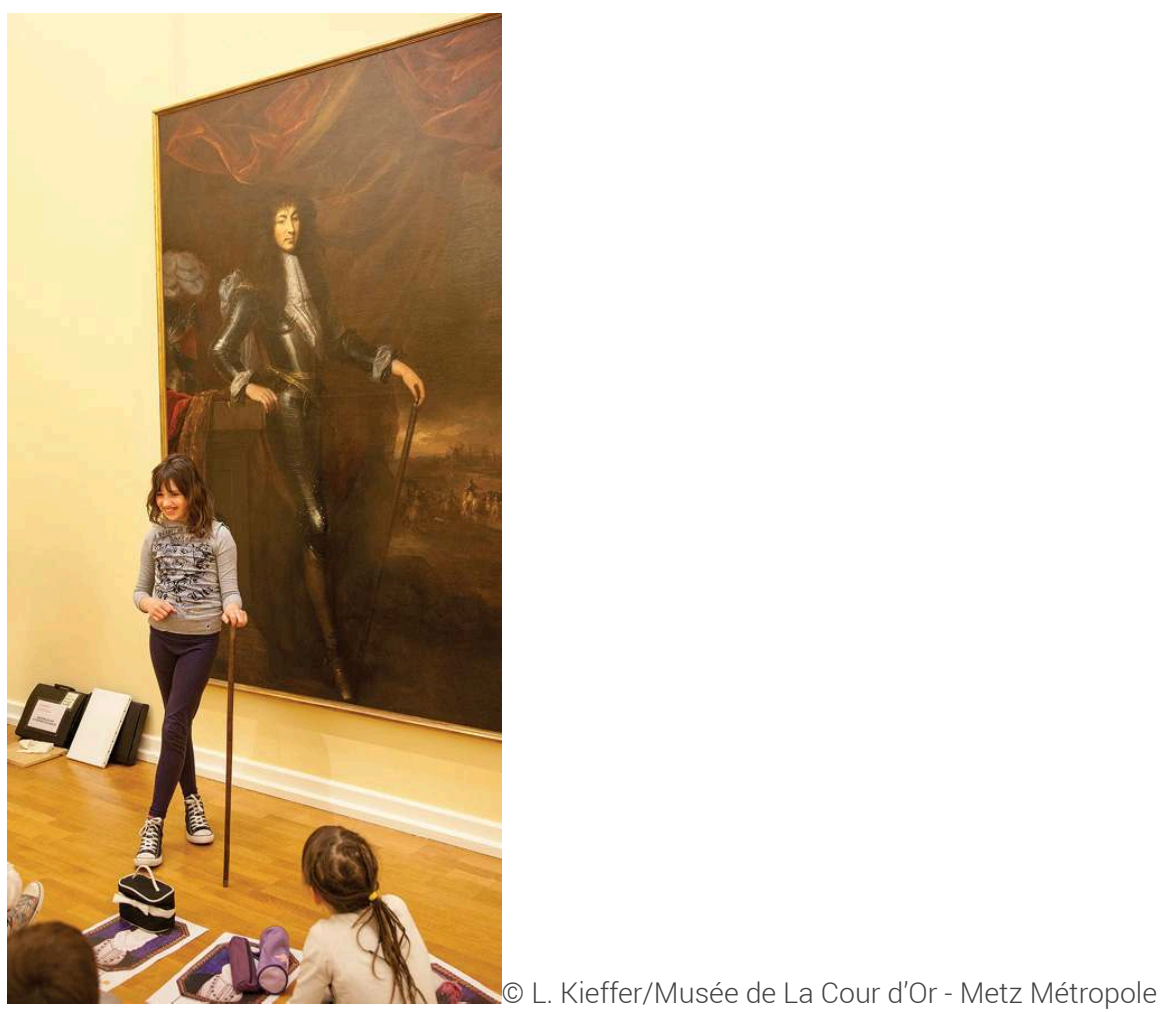




\section{Troisième étape : un ennemi puissant et l'épilogue}

Le protagoniste arrive à un point culminant de son aventure : il affronte, au choix, un minotaure caché dans un labyrinthe ou une sphinge perchée au sommet d'une montagne aux pentes abruptes (figures 7 et 8). Un jeu de labyrinthe papier ou une fiche d'énigmes tirées au hasard enrichissent la péripétie. Une fois le monstre vaincu les enfants achèvent leur histoire en décidant de la fin à lui donner (figure 9).

\section{Le lieu d'exposition comme catalyseur}

L'histoire est conçue de telle sorte que chaque groupe d'enfants a une probabilité quasinulle de vivre la même expérience que les autres. En fin d'année, tous les groupes comparent leurs récits, en classe. Le système offre donc une possibilité de restitution des séances et une relecture globale qui engage les enfants dans une démarche de partage et de communication.

\section{Le point de vue d'enseignantes de CE1-CE2 sur l'APC Le musée dont vous êtes le héros}

"Nous avons décidé de faire les APC au musée afin que les élèves découvrent un lieu (le musée) que très peu d'enfants connaissent. C'était l'occasion de sortir du contexte scolaire (la classe) et de profiter de l'expérience d'une médiatrice. Le projet permet aux enfants d'enrichir leur culture artistique, de découvrir un vocabulaire adapté, de laisser libre court à leur imagination et de créer une histoire (conte). Les séances sont donc axées sur l'amélioration du langage oral, la découverte de l'écrit pour les CE1, en particulier, et, par la production d'un récit. C'est un moment privilégié car nous venons avec un petit groupe d'élèves. Cela permet de solliciter chacun d'entre eux. Dans ce contexte différent, ils prennent plus facilement la parole. Certains se révèlent différents de leur attitude en classe. On les découvre sous un autre angle. Ils participent davantage et s'impliquent dans le projet. Ils attendent avec impatience la séance suivante. Une culture commune se crée dans un climat collaboratif. Le travail est réinvesti en classe sous forme d'une lecture de roman (sur le minotaure), d'un projet sur la mythologie, et en arts plastiques.

Nous avons un très bon retour de la part des parents qui nous disent que les enfants ont beaucoup aimé. Nous espérons ainsi que certains enfants reviendront avec leurs parents ».

Mme Pitelet et Mme Mengelr

Enseignantes de CE1-CE2 à l'école Camille Hilaire, Metz

Le musée de La Cour d'Or comporte 46 salles sur $6000 \mathrm{~m} 2$. De quoi donner l'impression de voyager dans de grands espaces. Nous jouons autour de la disposition des lieux et sur la muséographie/scénographie pour renforcer cet effet. Pour citer un exemple concret, afin d'escalader la montagne (imaginaire), les enfants vont passer par une série d'escaliers qui leur donnera la sensation de grimper réellement. Parvenus au sommet, tel Edipe de Gustave Moreau ${ }^{11}$, ils font face à la sphinge. Quoi de mieux pour comprendre la démarche du peintre et sa vision d'un héros humanisé et las de son voyage? 
$\mathrm{Au}$ final cette approche, quoi qu'elle fasse songer à la " gamification ${{ }^{12}}^{12}$ ou aux serious game dans les musées, s'appuie sur des outils « classiques » tels que le livre en version papier, avec du vocabulaire adapté, pouvant être lu par des enfants. Les compétences et les attendus travaillés sont ceux inscrits au Bulletin officiel pour cette tranche d'âge. Les enseignants sont une aide précieuse sur ce contenu et leur avis est toujours sollicité. Les documents leur sont présentés en amont. Des bilans intermédiaires permettent d'améliorer les détails au fur et à mesure. La notion de transversalité n'est pas oubliée : les dés font appel au calcul, le temps de voyage est compté.

Le groupe d'enfants débute son aventure avec un fragment de carte à compléter.

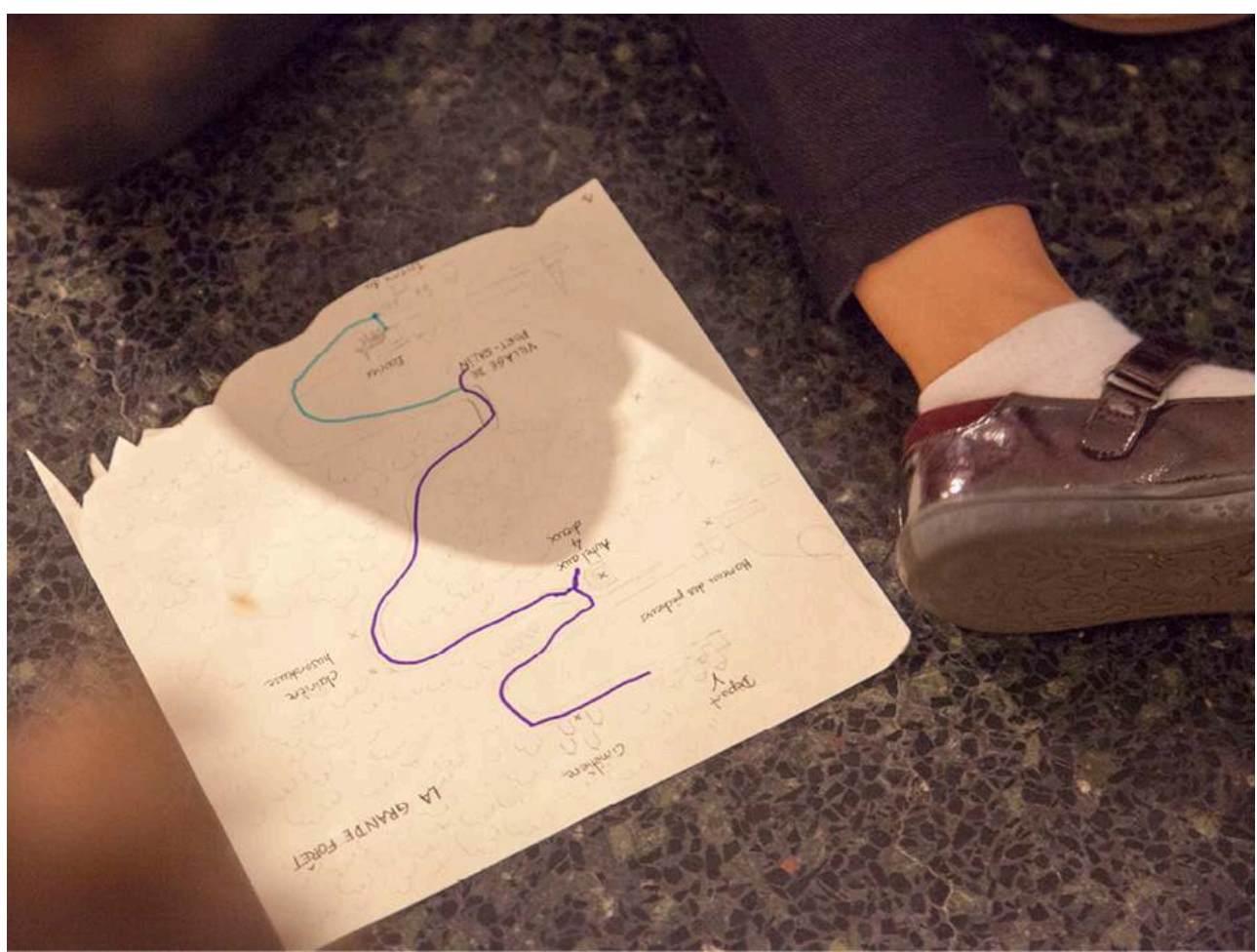

(c) L. Kieffer/Musée de La Cour d'Or - Metz Métropole

\section{Bilan et perspectives}

La phase d'expérimentation était gratuite sur le temps d'une année scolaire. Les élèves ont adhéré avec enthousiasme. Les groupes ont appris à coopérer. La principale victoire est, à notre sens, que les enfants ont vécu des moments marquants. La construction du conte a touché près de cinquante enfants de CE1-CE2, dont la moitié environ est revenue en 2020.

Ce test nous a également confortés dans l'idée de sortir des sentiers battus pour tenter de nouvelles expériences. Désormais nous avons pérennisé cette visite proposée au programme des activités à destination des scolaires. Notre méthodologie a fonctionné car la présence humaine du médiateur permet d'improviser. Dans le cadre des APC, nos places sont limitées et nous atteignons déjà la jauge maximale. En revanche, cette visite peut être déclinée sur des ateliers de vacances puisque ce sont aussi de petits groupes de six enfants. D'autres histoires seront sans doute créées sur ce modèle éprouvé. 


\section{NOTES}

1. Darras B. et Kindler A.-M. Le musée, l'école et l'éducation artistique, Publics et musée, 1998, $n^{\circ} 14$, Avignon, Actes Sud, pp. 15-37.

2. Gozlan A. Effet des actions de médiation culturelle sur les enfants et les adolescents, projet de recherche entre l'université de Picardie Jules Verne et l'université Nice Sophia Antipolis, Collectif, ministère de la Culture, 2018, 243 p.

3. Lagier J., De Barnier V. et Ayadi K. J'aime mon musée : la perception esthétique des enfants et leur rapport à l'Art. Management et avenir, $\mathrm{n}^{\circ} 78,2015$, pp. 41-57.

4. Van Dorpe A. et Scamps L. Des livrets pour accompagner les enfants dans les musées, la lettre de l'Ocim, 2008, n 120, pp. 4-10.

5. Socle commun de connaissances, de compétences et de culture. Bulletin officiel de l'Éducation nationale, n¹7, 23 avril 2015. Extrait du programme de l'école élémentaire cycle II : «L'élève parle, communique, argumente à l'oral de façon claire et organisée ; il adapte son niveau de langue et son discours à la situation, il écoute et prend en compte ses interlocuteurs".

6. Projet construit selon les préconisations du Rapport de la mission des musées du XXI siècle, et plus particulièrement la mesure qui vise à « adapter l'offre aux jeunes générations » (p. 18).

7. Les livres-jeux interactifs sont nés en 1960-1970, ils connaissent leur heure de gloire à partir de 1982 à la sortie Du sorcier de la montagne de feu de Steve Jackson et Ian Livingstone. Tombés dans l'oubli dans les années 1990, ils sont réédités en 2010, en littérature jeunesse.

8. Solinski B. À la marge de la lecture et du ludique : les livres-jeux. Sciences du jeu, nº 7, 2017, pp. 1-21.

9. La Maison d'Ailleurs : http://www.ailleurs.ch

10. Deny M. Le tournoi de tous les dangers. Paris : Nathan, 2015. 64 p.

11. Moreau G. Edipe voyageur, avant 1888, huile sur toile, 124 x $93 \mathrm{~cm}$. Collection du musée de La Cour d'Or, inv. 222.

12. Passebois-Ducrot J., Euzeby F., Machat S. et Lallement J. La gamification des dispositifs de médiation culturelle : quelle perception et quel impact sur l'expérience de visite ? Le cas de la corderie royale. La Rochelle : université de La Rochelle, 2015, 30 p.

\section{RÉSUMÉS}

C'est en s'appuyant sur les préconisations de professionnels et de chercheurs que le musée de La Cour d'Or-Metz Métropole a testé, dans le cadre des activités pédagogiques complémentaires, un mode de visite inédit - inspiré des Livres dont vous êtes le héros - qui a permis de définir une nouvelle méthodologie d'accueil du public scolaire.

\section{INDEX}

Mots-clés : exposition, expérience de visite, outil d'aide à la visite, outil pédagogique, public scolaire 
AUTEUR

DENISE DAVIN

Chargée des publics au musée de La Cour d'Or-Metz Métropole musee-accueil-publics@metzmetropole.fr 